\title{
THE STABILITY OF CERTAIN FUNCTIONAL EQUATIONS
}

\author{
JOHN A. BAKER
}

(Communicated by Kenneth R. Meyer)

\begin{abstract}
The aim of this paper is to prove the stability (in the sense of Ulam) of the functional equation:

$$
f(t)=\alpha(t)+\beta(t) f(\phi(t)),
$$

where $\alpha$ and $\beta$ are given complex valued functions defined on a nonempty set $S$ such that $\sup \{|\beta(t)|: t \in S\}<1$ and $\phi$ is a given mapping of $S$ into itself.
\end{abstract}

\section{INTRODUCTION}

Questions concerning the stability of functional equations seem to have originated with S. M. Ulam in the 1940s (see [2,3] and the survey paper of Hyers [4]). One of the first assertions to be proved in this direction is the following result, essentially due to Hyers, that answered a question of Ulam.

Theorem. Suppose $S$ is an additive semigroup, $E$ is a Banach space, $f: S \rightarrow E$, $\delta>0$, and

$$
\|f(x+y)-f(x)-f(y)\| \leq \delta \text { for all } x, y \in S .
$$

Then there is a unique function $a: S \rightarrow E$ such that

$$
a(x+y)=a(x)+a(y) \text { for all } x, y \in S,
$$

and

$$
\|f(x)-a(x)\| \leq \delta \quad \text { for } x \in S .
$$

This assertion is usually summarized by saying that the Cauchy functional equation (2) is stable (in the sense of Ulam).

The proof proceeds as follows. First note that

$$
\|f(2 x) / 2-f(x)\| \leq \frac{\delta}{2} \text { for all } x \in S .
$$

Received by the editors January 2, 1990.

1980 Mathematics Subject Classification (1985 Revision). Primary 39B10, 26D20; Secondary 39B70, 47H10.

Key words and phrases. Functional equations, stability, fixed points. 
Then one proves, by induction, that $\left\{f\left(2^{n} x\right) / 2^{n}\right\}_{n=1}^{\infty}$ is a Cauchy sequence in $E$. Denoting its limit by $a(x)$, one then proves that $(2)$ and $(3)$ hold. The same idea can be used to prove the stability of the equation:

$$
f(2 x) / 2=f(x) .
$$

In fact the same technique can be used to prove the

Proposition. Suppose $S$ is a nonempty set, $\phi: S \rightarrow S, E$ is a Banach space, $\lambda$ is a scalar, $|\lambda|<1, \delta>0$, and $f: S \rightarrow E$ such that

$$
\|\lambda f(\phi(x))-f(x)\| \leq \delta \text { for all } x \in S .
$$

Then there exists a unique $h: S \rightarrow E$ such that

$$
\lambda h(\phi(x))=h(x) \text { for all } x \in S,
$$

and

$$
\|f(x)-h(x)\| \leq \delta /(1-|\lambda|) \quad \text { for all } x \in S .
$$

Proof. Let $\phi^{k}$ denote the $k$ th iterate of $\phi$ for $k=0,1,2, \ldots$. Thus $\phi^{0}(x)=$ $x, \phi^{1}(x)=\phi(x), \phi^{2}(x)=\phi(\phi(x))$, etc. for all $x \in S$.

By induction, it follows from (6) that

$$
\left\|\lambda^{k+1} f\left(\phi^{k+1}(x)\right)-\lambda^{k} f\left(\phi^{k}(x)\right)\right\| \leq|\lambda|^{k} \delta \text { for all } x \in S \text { and } k=0,1,2, \ldots
$$

Hence, if $m$ and $n$ are nonnegative integers and $m<n$, then

$$
\begin{aligned}
\left\|\lambda^{n} f\left(\phi^{n}(x)\right)-\lambda^{m} f\left(\phi^{m}(x)\right)\right\| & \leq\left(|\lambda|^{n-1}+\cdots+|\lambda|^{m}\right) \delta \\
& \leq \frac{|\lambda|^{m} \delta}{1-|\lambda|} \text { for all } x \in S .
\end{aligned}
$$

Thus $\left\{\lambda^{n} f\left(\phi^{n}(x)\right)\right\}_{n=1}^{\infty}$ is a Cauchy sequence in $E$ for each $x \in S$. Let $h(x)=$ $\lim _{n \rightarrow \infty} \lambda^{n} f\left(\phi^{n}(x)\right)$ for $x \in S$. Then (7) clearly holds.

If we choose $m=0$ in (9) we find that

$$
\left\|\lambda^{n} f\left(\phi^{n}(x)\right)-f(x)\right\| \leq \delta /(1-|\lambda|) \quad \text { for all } x \in S,
$$

and all $n=1,2, \ldots$, and this implies $(8)$.

Most known stability theorems for functional equations concern functional equations in "several variables" in the sense of Aczél [1]. Equation (2) is the best-loved representative of this class. Equation (7), whose stability we have just proved, is often referred to as a functional equation in a "single variable." Such equations are the subject of the book by Kuczma [5]. The aim of this paper is to generalize the above proposition by proving a stability result for the general functional equation:

$$
f(t)=F(t, f(\phi(t)) .
$$

We refer the reader to [5] for numerous results and references concerning this equation and particular cases thereof.

\section{MAIN RESULTS}

Our analysis is based on the following variant of Banach's fixed point theorem that is likely included among the thousands of published generalizations thereof. 
Theorem 1. Suppose $(Y, \rho)$ is a complete metric space and $T: Y \rightarrow Y$ is a contraction (for some $\lambda \in[0,1), \rho(T(x), T(y)) \leq \lambda \rho(x, y)$ for all $x, y \in Y)$. Also suppose that $u \in Y, \delta>0$, and

$$
\rho(u, T(u)) \leq \delta .
$$

Then there exists a unique $p \in Y$ such that $p=T(p)$. Moreover, $\rho(u, p) \leq$ $\delta /(1-\lambda)$.

Proof. Define a sequence $\left\{y_{n}\right\}_{n=0}^{\infty}$ in $Y$ by decreeing that $y_{0}=u$ and $y_{n+1}=$ $T\left(y_{n}\right)$ for all $n=0,1,2, \ldots$. According to the well-known proof of Banach's fixed point theorem, $\left\{y_{n}\right\}_{n=1}^{\infty}$ converges, say to $p, p$ is the unique fixed point of $T$, and

$$
\rho\left(y_{n}, p\right) \leq \frac{\lambda^{n}}{1-\lambda} \rho\left(y_{1}, y_{0}\right) \text { for } n=0,1,2, \ldots
$$

Thus

$$
\rho(u, p) \leq \rho(u, T(u))+\rho(T(u), p) \leq \delta+\rho(T(u), T(p)) \leq \delta+\lambda \rho(u, p)
$$

so that $\rho(u, p) \leq \delta /(1-\lambda)$.

Theorem 2. Suppose $S$ is a nonempty set; $(X, d)$ is a complete metric space; $\phi: S \rightarrow S, F: S \times X \rightarrow X, 0 \leq \lambda<1$; and

$$
d(F(t, u), F(t, v)) \leq \lambda d(u, v) \quad \text { for all } t \in S \text { and all } u, v \in S .
$$

Also suppose that $g: S \rightarrow X, \delta>0$, and

$$
d(g(t), F(t, g(\phi(t)))) \leq \delta \quad \text { for all } t \in S .
$$

Then there is a unique function $f: S \rightarrow X$ such that

$$
f(t)=F(t, f(\phi(t))) \text { for all } t \in S,
$$

and

$$
d(f(t), g(t)) \leq \delta /(1-\lambda) \quad \text { for all } t \in S .
$$

Proof. Let $Y=\{a: S \rightarrow X \mid \sup \{d(a(t), g(t)) \mid t \in S\}<+\infty\}$. For $a, b \in Y$ define $\rho(a, b)=\sup \{d(a(t), b(t)) \mid t \in S\}$. Then $g \in Y, \rho$ is a metric on $Y$, and convergence with respect to $\rho$ means uniform convergence on $S$ with respect to $d$. Moreover, the completeness of $X$ with respect to $d$ implies the completeness of $Y$ with respect to $\rho$.

For $a \in Y$ define $T(a): S \rightarrow X$ by

$$
(T(a))(t)=F(t, a(\phi(t))) \quad \text { for } t \in S .
$$

Then $T$ maps $Y$ into $Y$. If $a, b \in Y$ then for all $t \in S$,

$$
\begin{aligned}
d((T(a))(t),(T(b))(t)) & =d(F(t, a(\phi(t))), F(t, b(\phi(t)))) \\
& \leq \lambda d(a(\phi(t)), b(\phi(t))) \\
& \leq \lambda \rho(a \circ \phi, b \circ \phi) \\
& \leq \lambda \rho(a, b) .
\end{aligned}
$$


Thus,

$$
\rho(T(a), T(b)) \leq \lambda \rho(a, b) \text { for all } a, b \in Y .
$$

But (10) means that $\rho(g, T(g)) \leq \delta$. Hence, according to Theorem 1 , there is a unique $f$ in $Y$ such that $f=T(f)$ and $\rho(g, f) \leq \delta /(1-\lambda)$. That is, (11) and (12) hold.

We will now use Theorem 2 to deduce stability assertions concerning the functional equation:

$$
f(t)=\alpha(t)+\beta(t) f(\phi(t)) .
$$

Theorem 3. Suppose $S$ is a nonempty set; $E$ is a real (or complex) Banach space; $\phi: S \rightarrow S, \alpha: S \rightarrow E, \beta: S \rightarrow \mathbb{R}($ or $\mathbb{C}), 0 \leq \lambda<1 ;$ and $|\beta(t)| \leq \lambda$ for all $t \in S$. Also suppose that $g: S \rightarrow E, \delta>0$, and

$$
\|g(t)-\{\alpha(t)+\beta(t) g(\phi(t))\}\| \leq \delta \text { for all } t \in S \text {. }
$$

Then there exists a unique function $f: S \rightarrow E$ such that

$$
f(t)=\alpha(t)+\beta(t) f(\phi(t))
$$

and

$$
\|f(t)-g(t)\| \leq \delta /(1-\lambda) \text { for all } t \in S .
$$

Proof. The result follows from Theorem 2 by letting

$$
F(t, x)=\alpha(t)+\beta(t) x \text { for }(t, x) \in S \times E .
$$

Similarly we can prove

Theorem 4. Suppose $S$ is a nonempty set; $A$ is a Banach algebra; $\phi: S \rightarrow S$, $\alpha: S \rightarrow A, \beta: S \rightarrow A ; 0 \leq \lambda<1,\|\beta(t)\| \leq \lambda$ for all $t \in S, g: S \rightarrow A$, $\delta>0$; and (13) holds. Then there is a unique function $f: S \rightarrow A$ satisfying (14) and (15).

\section{ACKNOWLEDGMENT}

A referee has noted that our proposition is a particular case of Theorem 1 and Corollary 3 of G. L. Forti, An existence and stability theorem for a class of functional equations, Stochastica 4 (1980), 23-30.

\section{REFERENCES}

1. J. Aczél, Lectures on functional equations and their applications, Academic Press, New York, 1966.

2. Michael Albert and J. A. Baker, Functions with bounded nth differences, Ann. Polon. Math. 42 (1983), 93-103.

3. J. A. Baker, The stability of the cosine equation, Proc. Amer. Math. Soc. 80 (1980), 411-416.

4. D. H. Hyers, The stability of homomorphisms and related topics, Global Analysis-Analysis on Manifolds (T. M. Rassias, ed.), Teubner-Texte zur Mathematik, Band 57, Teubner Verlagsgesellschaft, Leipzig, 1983, pp. 140-153.

5. M. Kuczma, Functional equations in a single variable, Monographs Math., vol. 46, PWN, Warszawa, 1968.

Department of Pure Mathematics, University of Waterloo, Waterloo, Ontario, N2L 3G1 CANADA 